\title{
Comparison of inductances of brake resistor blocks for electric vehicles with spiral and tape resistive elements
}

\author{
Anton Timofeev ${ }^{1, *}$ and Alexander Shapovalov ${ }^{2}$ \\ ${ }^{1}$ Research and production company «Etna plus », 410040, Vishnevaya str. 11, Saratov, Russia \\ ${ }^{2}$ Saratov State University, Physical Department, 4120012. Astrakhanskaya str. 83, Saratov, Russia
}

\begin{abstract}
The paper presents the calculated and experimental results of a comparison of two main types of brake resistor blocks structures. A mathematical model is given for finding the inductance of resistor blocks with spiral and tape resistive elements. Approaches had been carried out to determine the most advantageous configurations of the resistive unit in terms of minimizing its inductance value.
\end{abstract}

\section{Introduction}

Public transport vehicles in developed countries are switching to electric traction everywhere. It is advisable to slow down a weighing tens of tons vehicle in service modes, using its electric motor, which goes into the generation mode. It's good from the point of view of reliable contact with the road and the wheel brakes resource. In modern vehicles powered by the contact network, as well as electric buses, most of the braking energy is recycled back to the battery or to the contact network, but this is not always possible (the battery can be charged, and the network does not have powerful consumers at this moment). That's why it is necessary to use the so-called brake resistors - powerful electrical resistive devices that can dissipate thermal power of hundreds or even thousands of kilowatts while tens of seconds. At the same time, these devices must have stable performance, be electrically safe and protected from temperature jumps, when water (precipitation) enters inside them, from electrical breakdowns of air at high humidity and temperature.

As a rule, modern devices of this type work in conjunction with inverter electronic motor controllers operating in a pulsed mode - with pulse width modulation (PWM) load switching with a reference frequency of the order of several tens of $\mathrm{kHz}$. Dew to this circumstance, a current in the form of rectangular pulses with a duration of less than $1 \mathrm{~ms}$ and an amplitude of hundreds of amperes flows across the braking resistors [1]. In such conditions, for the overall reliability, efficiency and minimization of interference, caused by such a "network-controller-motor-brake resistor" system, it is desirable to have the inductance value of the resistor blocks as little, as possible.

\footnotetext{
* Corresponding author: anton timofeev@list.ru
} 
At present, the most common in the industry are 2 basic designs of the resistive elements including in these blocks - compositions of spirals (Figs. 1), formed from a special alloy wire with high resistivity [2], and sets (Figs. 2.) of flat plates (possibly with molded elements to increase rigidity and their fastening), which, due to the need to obtain a certain ohmic resistance of the block at the required current section and heat dissipating surface area, become most like a tape [3].

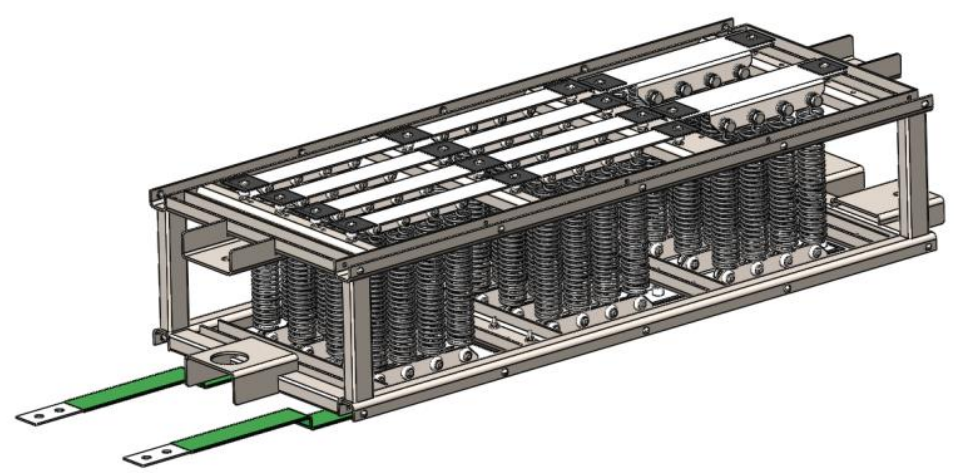

Fig. 1. Appearance of the block with spiral resistive elements.

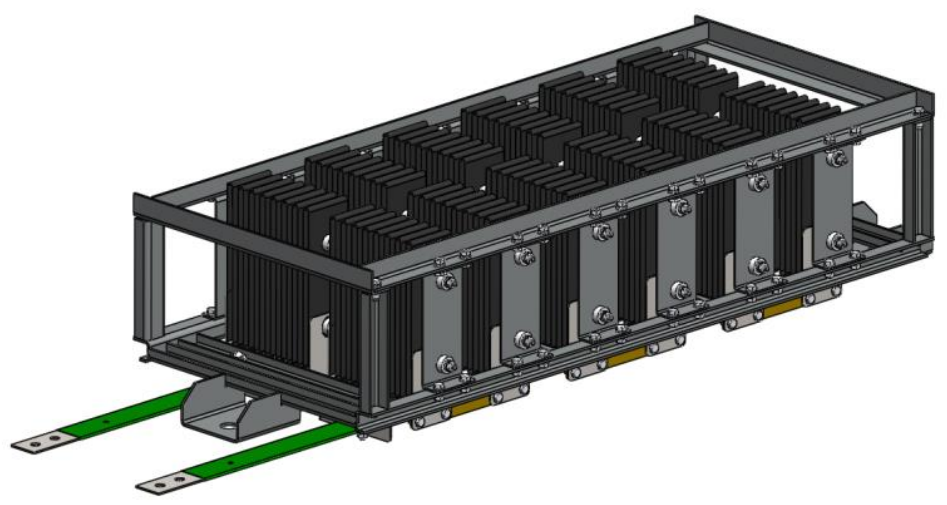

Fig. 2. Appearance of the block with resistive elements produced of a flat ribbon designed in the form of a meander.

The present work is devoted to comparing the characteristics of these designs from various points of view. The main area of research in it is the determination and comparison of the inductances of two basic designs of the resistor block with almost identical operational characteristics (identical resistances, similar in value to heat dissipation ability, with almost equal weight and dimensions of the resistive elements compositions). One of these blocks was made with spiral resistive elements, and the other - with resistive elements produced of a flat ribbon designed in the form of a meander, both blocks were designed from the same materials (either fechral or heat-resistant stainless steel).

\section{Comparison of the inductance of spiral and tape resistive elements}

In the course of conducted by the authors research, the calculation of a single spiral resistive element (Fig. 3) inductance $L$ was initially undertaken. This value was determined from the relation, obtained on the basis of [4]: 


$$
L=\mu_{0} N^{2} S / l=\pi R^{\prime 2} \mu_{0} N^{2} / l
$$

where $N$ - is the number of turns of the spiral, $S$ - is the cross-sectional area of the spiral, $l$ is the length of the spiral, $R^{\prime}$ - is the average radius of the spiral turn, $\mu_{0}$ - is the magnetic constant $\left(\mu_{0}=4 \pi * 10-7 \mathrm{H} / \mathrm{m}\right)$.

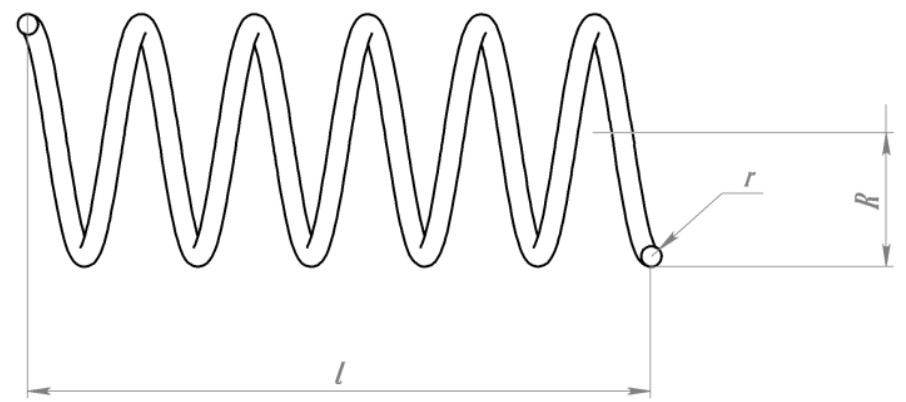

Fig. 3. Appearance of a single spiral resistive element.

In this case, the average radius of the spiral is equal to $R^{\prime}=(R-r)$, where $R$ - is the outer radius of the spiral, $r$ - is the radius of the wire from which the spiral is wound, it is taken as the value $R$ in the ratio (1). From relation (1), in particular, it follows the physically understandable conclusion, that when the denser of the spiral winding is increasing, the inductance will increase also. In our case, the spiral pitch $\mathrm{h}$ is approximately equal 2 (two) diameters of the wire from which it is wound $(h \approx 4 r)$, which can significantly reduce the inductance of the spirals and the entire composition of the spiral resistive elements underlying the design of the resistor block.

For a single spiral, used in one of the mentioned blocks $\left(R^{\prime}=29 \mathrm{~mm}, r=1,5 \mathrm{~mm}, l=\right.$ $159 \mathrm{~mm}, N=26$ ), calculation according to (1) gives the value $L=2,2 \mathrm{H}$.

If a non-magnetic material is selected for the manufacturing of spiral resistive elements (for example, heat-resistant stainless steel), formula (1) allows rather accurately calculate its inductance value and obtain the results, got above. If a soft magnetic material (for example, fechral) is used, then its magnetic permeability should be taken into account (of course, taking into account the fill factor $\mathrm{k}$ (relation of the volume, filled with this material to the total volume of the spiral resistive element).

If the magnetic permeability of the material of the wire, from which the spiral is wound, is $\mu$ and the fill factor $\mathrm{k}$ can be approximately written as

$$
k=\pi r^{2}\left(2 \pi R^{\prime}\right) / \pi R^{\prime} 2 h=2 \pi r^{2} / R^{\prime} h
$$

where in the numerator is the volume occupied by the spiral wire on one of its turns is recorded, and in the denominator - the volume of the entire turn itself.

Then the inductance obtained from formula (1) should be multiplied by the coefficient $\mu_{e f}$, where

$$
\mu_{e f}=2 \pi r^{2} \mu / R^{\prime} h
$$

and, accordingly, the inductance of the spiral resistive element will be

$$
L=2 \mu_{0} \mu \pi^{2} r^{2} R^{\prime} N^{2} / h l
$$

The structure under consideration (Fig. 1) consists of connected in series assemblies, each of which includes $4(m=4)$ spirals, connected in parallel. The resistor block includes 12 such assemblies connected in series $(k=12)$. The total resistance of such unit was $1,4 \mathrm{Ohms}$. 
In the present work, the inductance was calculated without taking into account the interaction of the magnetic fields of the spirals with each other (neglecting their mutual inductance), under the assumption of a weak effect of elements removed from each other. Subsequent experiments showed that the effect of changing the distance between adjacent elements on the inductance of the entire assembly reaches units of percent only when the centers of the spirals come closer to a distance less than 3 spiral radii, which is not used in real designs of resistive elements.

In this regard, the total inductance of the resistor block $L_{\Sigma}$ was calculated by the formula

$$
L_{\Sigma}=k L / m
$$

In this calculation, the total value of the resistor block inductance, obtained by spiral resistive elements made of non-magnetic material, is $L_{\Sigma}=6,63 \mu \mathrm{H}$. When they are made of magnetic material (fechral), the inductance increases and amounts to $L_{\Sigma}=35,4 \mu \mathrm{H}$.

Important characteristics for the designing of such devices are inductance per unit volume $L_{v}$ and inductance per unit length $L_{l}$. For a single spiral, $L_{v}$ can be determined from the formula

$$
L_{v}=\mu_{0} N^{2} / l^{2}=\mu_{0} n^{2}
$$

where $n$ - is the number of turns per unit length of the spiral, and inductance per unit length can be calculated by the formula

$$
L_{l}=\mu_{0} \pi R^{\prime} \mu_{0} N /(2 \pi d)
$$

For comparison with the obtained parameters of a block of resistors with spiral resistive elements, it seemed necessary to calculate the inductance of a similar block with tape resistive elements. For this aim, on the basis of [4], a mathematical model was created to find the inductance of this configuration of the resistive elements.

The design under consideration is based on a flat ribbon bent in the form of a meander (Fig. 4) from a material with high resistivity (fechral or heat resistant stainless steel).

Neglecting the influence of the mutual inductances of the long sections (bands) of the meander, we consider their total inductance as the sum of the inductances of each of the bands (we take into account only the influence of the two nearest bands with current). In this case, the total inductance formula has the same form

$$
L=N l L_{0}=\frac{N l \mu_{0}}{4 w /(h-t)+t / h}
$$

where $L_{0}$ - is the linear inductance, $N$ - is the number of long sections of the meander sequentially connected (using the "short" sections), $w$ - is the width and $t$ - is the thickness of the tape, $h$ - is the distance between the centers of the strips in the long sections (halfperiod of the "meander"), $l$ - is the length of the long section (the meander span).

If (based on [4]) we take into account the inductance of the end transitions ("short" sections of the "meander"), then for one such section

$$
L_{T}=\frac{\mu_{0} l_{0}}{2 \pi} \ln \left(l_{0} /(w+t)+1 / 2\right)
$$

where $d$ - is the meander period $(d=2 h)$.

Hence the total inductance of one resistive element is

$$
L=N\left(l L_{0}+L_{T}\right)=\frac{N l \mu_{0}}{4 w /(h-t)+t / h}+\frac{N \mu_{0} l_{0}}{2 \pi} \ln \left(l_{0} /(w+t)+1 / 2\right)
$$




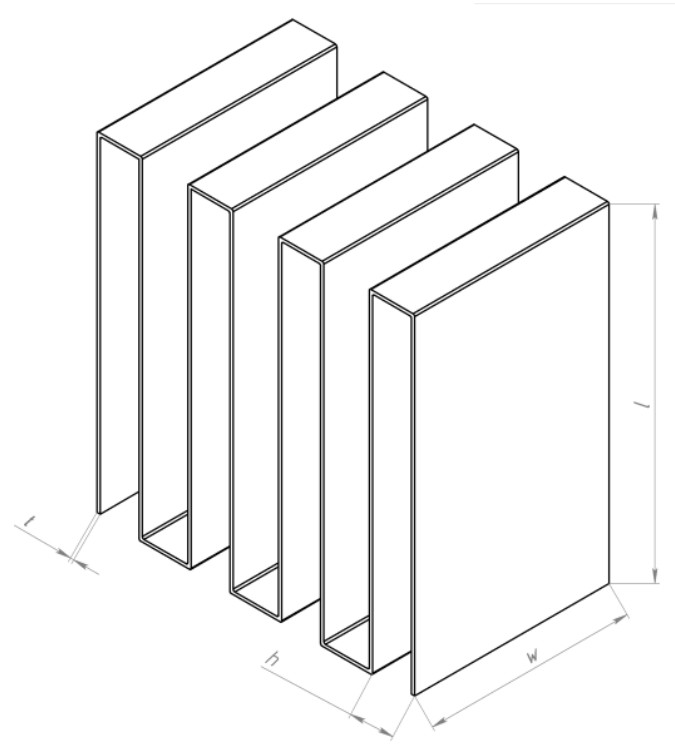

Fig. 4. Appearance of a meander resistive element.

If the nonmagnetic material (for example, heat-resistant stainless steel) is chosen for the manufacturing of "meander" resistive elements, formula (10) allows to calculate its inductance quite accurately (the calculation results are given below in comparison with the obtained experimental data). If a soft magnetic material (for example, fechral) is used, then its magnetic permeability should be taken into account (of course, taking into account the fill factor k - describing relative volume of the "meander", filled with this material).

If the magnetic permeability of the tape is $\mu$, and the fill factor $k=w t /(w t+d w)=t / d$ then the inductance obtained from formula (10) should be also multiplied by the coefficient $\mu_{e f}$, where

$$
\mu_{e f}=\left[\frac{w t(l+h)}{l w h}\right] \mu=\frac{\mu t(l+h)}{l h}
$$

and, accordingly, the meander inductance will be

$$
L=N\left(l L_{0}+L_{T}\right)=\mu_{0} \mu \frac{N t(l+h)}{h}\left[\frac{1}{4 w /(h-t)+t / h}+\frac{l_{0} / l}{2 \pi} \ln \left(l_{0} /(w+t)+1 / 2\right)\right]
$$

Naturally, with a decrease in the thickness of the tape relative to the meander period, the influence of $\mu_{e f}$ decreases, but this is often unacceptable based on the thermophysical analysis of the system and the need to ensure the mechanical strength of the plates.

The total length of the ribbon in the meander is $l_{F}=N(2 l+d)$. Inductance per unit of length (excluding the "magnetic" coefficient $\mu_{e f}$ - for a resistive element made of nonmagnetic material)

$$
L_{l}=\frac{l \mu_{0}}{2 l+d}(1-t / d+t \mu / d)\left[\frac{1}{4 w /(h-t)+t / h}+\frac{l_{0} / l}{2 \pi} \ln \left(l_{0} /(w+t)+1 / 2\right)\right] \approx \frac{\mu_{0}}{2} \frac{(1-t / d+t \mu / d)}{4 w /(h-t)+t / h} \approx \mu_{0} \frac{(1-t / d+t \mu / d)}{8 w / h}
$$

The volume of the meander resistive element is $V=N d l w$. Inductance per unit of volume (excluding the "magnetic" coefficient $\mu_{e f}$ - for a resistive element made of non-magnetic material) 


$$
L_{v}=\frac{\mu_{0}}{4 w^{2} /(h-t)+t / h}+\frac{\mu_{0} l_{0} / l}{2 \pi w} \ln \left(l_{0} /(w+t)+1 / 2\right) \approx \frac{\mu_{0}}{4 w^{2} / h}+\frac{\mu_{0} l_{0} / l}{2 \pi w} \ln \left(l_{0} /(w+t)+1 / 2\right)
$$

Based on the obtained results of the calculations, and in order to verify the applicability of the written and used calculation formulas, the inductance values were measured for all the considered variants of resistor blocks, designed using both spiral and tape resistive elements (see tables 1,2). The measurement of the inductance was carried out using a standard calibrated inductance meter (RLC AKIP-6109).

Table 1. Comparison of calculated data and experimental results for a block with spiral resistive elements designed from fechral.

\begin{tabular}{|c|c|c|}
\hline Parameter Name & $\begin{array}{c}\text { Calculated } \\
\text { data }\end{array}$ & $\begin{array}{c}\text { Experimental } \\
\text { results }\end{array}$ \\
\hline Single helix inductance, $\mu \mathrm{H}$ & 11,1 & 11,8 \\
\hline Inductance of a block of 4 parallel-connected spirals, $\mu \mathrm{H}$ & 2,75 & 2,95 \\
\hline $\begin{array}{c}\text { Inductance 12 series-connected blocks of 4 parallel-connected } \\
\text { spirals each, } \mu \mathrm{H}\end{array}$ & 33,1 & 35,4 \\
\hline
\end{tabular}

Table 2. Comparison of calculated data and experimental results for a block with tape resistive elements designed from fechral.

\begin{tabular}{|c|c|c|}
\hline Parameter Name & $\begin{array}{c}\text { Calculate } \\
\text { d data }\end{array}$ & $\begin{array}{c}\text { Experimental } \\
\text { results }\end{array}$ \\
\hline Inductance of a single meander composition, $\mu \mathrm{H}$ & 0,33 & 0,35 \\
\hline Inductance 6 of series-connected "meander compositions", $\mu \mathrm{H}$ & 1,98 & 2,1 \\
\hline
\end{tabular}

From the presented data, it is possible to judge about a sufficiently high degree of agreement between the calculated and experimental data (the discrepancy is not more than $7 \%$, which seems quite correct for engineering calculations, especially due to the fact, that the main parameter for these devices - is their resistance, and the inductance values are just only a limitation).

As for the main aim of this work - comparing the inductance of blocks with different types of resistive elements - the comparative calculations showed that for blocks of brake resistors with the same system of parameters (resistance, heat dissipation ability, etc.), the blocks, based on tape resistive elements, have something about 17 times lower inductance than blocks with spiral resistive elements. An experimental study of the two indicated types of blocks showed approximately the same results.

\section{Conclusion}

The design of brake resistor blocks for various applications represents a wide field for research, since before it sets the task of reliable operation with limited dimensions, weight and economic resources. The requirements for ensuring compatibility with modern motor inverter controllers regarding inductance of these blocks also impose their limitations.

A practical study of these structures showed that the resistor blocks, using the tape resistive elements of the corresponding configuration, are also the most promising due to their manufacturability, high heat dissipation ability and compactness.

Using these blocks, it is significantly easier (in comparison with blocks built on spiral resistive elements) to implement quite stringent requirements to minimize their inductance to allowed values for the efficient operation of the electric drives with which they are used in vehicle electrical systems.

In this regard, the approaches developed in this work to determine the most advantageous configurations of the resistive unit performed on tape elements in terms of minimizing the inductance value are of great practical importance, just as the methods for their calculation and modeling. 


\section{References}

1. N.P. Vintonyak, V.D. Semenov, V.A. Kabirov et al. Estimation of the value of parasitic inductances of busbars in the power supply of the test complex for loading DC circuit breakers // TUSUR Reports, Volume 19, No. 4. P. 129-132 (2016)

2. Pat. Utility Model No. PM145666 Ros. Federation. / Resistor block for public transport / Kozlov A.V., Timofeyev A.I.; patent holder - NPF Etna. - declared. 07.08.2014, issue 08.21.2014, publ. 10.10.2014, Bull. № 21 .

3. Pat. Utility Model No. PM186566 Ros. Federation. Power resistor block / Timofeev A.I., Mashkov I.V. and etc.; Patent owner - NPF Etna Plus, declared 10.10.2018, issue 01.24.2019, publ. 01.24.2019, Bull. № 3.

4. Kalantarov P.L., Zeitlin L.A. Inductance calculation. - L.: Energoatomizdat, 488 p. (1986) 